\title{
Increased homocysteine levels impair reference memory and reduce cortical levels of acetylcholine in a mouse model of vascular cognitive impairment.
}

\author{
Kevin Dam, MSc ${ }^{a}$, Martina Füchtemeier, PhD ${ }^{a, b}$, Tracy D. Farr, PhD ${ }^{a}$, Philipp Boehm- \\ Sturm, PhD ${ }^{a}$, Marco Foddis ${ }^{a}$, Ulrich Dirnagl,MD ${ }^{a, b}$, Olga Malysheva PhD ${ }^{c}$, Marie A. \\ Caudill $\mathrm{PhD}^{\mathrm{c}}$, Nafisa M. Jadavji, PhD ${ }^{\mathrm{a}}$ \\ a Department of Experimental Neurology, Center for Stroke Research, \\ Berlin, Charité University Medicine Berlin, Germany \\ ${ }^{\mathrm{b}}$ German Center for Neurodegenerative Diseases (DZNE) Berlin Site, Berlin, Germany \\ ${ }^{c}$ Division of Nutritional Sciences, Cornell University, Ithaca, USA
}

\section{Corresponding Author:}

Nafisa M. Jadavji, PhD

Department for Experimental Neurology

Charité University Medicine Berlin

10117 Berlin GERMANY

Phone +49(0)30450560336

Fax +49 (0)30 450560974

E-mail: nafisa.jadavji@mail.mcgill.ca

Running title: Impact of elevated levels of homocysteine on $\mathrm{VCl}$

\section{KEYWORDS}

vascular cognitive impairment, chronic hypoperfusion, methylenetetrahydrofolate reductase, folic acid, homocysteine, choline, matrix metallopeptidase- 9 , tau 


\section{ABSTRACT}

Folates are B-vitamins that are vital for normal brain function. Deficiencies in folates either genetic (methylenetetrahydrofolate reductase, MTHFR) or dietary intake of folic acid result in elevated levels of homocysteine. Clinical studies have shown that elevated levels of homocysteine (Hcy) may be associated with the development of dementia, however this link remains unclear. The purpose of this study was to evaluate the impact of increased Hcy levels on a mouse model of vascular cognitive impairment ( $\mathrm{VCl})$ produced by chronic hypoperfusion. Male and female $\mathrm{Mthfr}^{+/+}$and $\mathrm{Mthfr}^{+/-}$mice were placed on either control (CD) or folic acid deficient (FADD) diets after which all animals underwent microcoil implantation around each common carotid artery or a sham procedure. Post-operatively animals were tested on the Morris water maze (MWM), Ymaze, and rotarod. Animals had no motor impairments on the rotarod, Y-maze, and could learn the location of the platform on the MWM. However, on day 8 of testing of MWM testing during the probe trial, $\mathrm{Mthfr}^{+/-}$FADD microcoil mice spent significantly less time in the target quadrant when compared to $\mathrm{Mthfr}^{+/-} \mathrm{CD}$ sham mice, suggesting impaired reference memory. All FADD mice had elevated levels of plasma homocysteine. $\mathrm{MRI}$ analysis revealed arterial remodeling was in $\mathrm{Mthfr}^{+/-}$microcoil mice not $\mathrm{Mthfr}^{+/+}$ mice. Acetylcholine and related metabolites were reduced in cortical tissue because of microcoil implantation and elevated levels of homocysteine. Deficiencies in folate metabolism resulting in increased Hcy levels yield a metabolic profile that increases susceptibility to neurodegeneration in a mouse model of VCl. 


\section{INTRODUCTION}

Vascular cognitive impairment $(\mathrm{VCl})$ is a type of dementia with a vascular origin. It is the second most common form of dementia and cognitive decline after Alzheimer's disease

1. The clinical presentation of $\mathrm{VCl}$ varies, but a majority of patients have some degree of cognitive decline ${ }^{1}$. Currently, the pathophysiology of $\mathrm{VCl}$ remains unknown and there are no standard treatments for affected individuals ${ }^{2}$. Therefore, understanding the basic mechanisms of $\mathrm{VCl}$ will enable potential therapies to be developed.

Nutrition is a modifiable risk factor in the development of $\mathrm{VCl}^{3}$. Folates are $\mathrm{B}$-vitamins that are essential for normal brain function because of their role in nucleotide synthesis, DNA repair and methylation. Furthermore, these vitamins are involved in metabolizing a neurotoxic amino acid called homocysteine. Epidemiological studies have linked elevated levels of homocysteine (Hcy) to increased vascular diseases ${ }^{4}$. More recently deficiencies in folate metabolism and resultant elevated levels of Hcy have been associated with onset or development of dementia ${ }^{5,6}$. A meta-analysis reported a positive association between increased levels of serum Hcy and incidence of dementia ${ }^{6}$, however, the data still remains controversial ${ }^{7}$.

Methylenetetrahydrofolate reductase (MTHFR) is an enzyme that breaks folates down into usable forms; it contributes a methyl group to remethylate Hcy, therefore, reducing levels of Hcy. A common polymorphism in Mthfr $(677 \mathrm{C} \rightarrow \mathrm{T})$ is present in $5-15 \%$ of North American and European populations, resulting in elevated levels of plasma $\mathrm{Hcy}^{8}$, and has been associated with the development of vascular disease ${ }^{9}$. A mouse model for the human MTHFR polymorphism has been developed and MTHFR-deficient $\left(\mathrm{Mthfr}^{+/}\right)$mice 
have reduced enzyme activity and elevated levels of Hcy when compared to wild-type controls and model the Mthfr $(677 \mathrm{C} \rightarrow \mathrm{T})$ polymorphism observed in humans ${ }^{10}$.

Animal models that mimic aspects of $\mathrm{VCl}$ have been developed to understand the pathophysiology of the disease ${ }^{11}$. Chronic hypoperfusion, a mouse model of $\mathrm{VCl}$, involves wrapping microcoils around the common carotid arteries and reduces blood flow

to the brain ${ }^{12}$. Using this model, previous work has reported impairments in spatial working memory in the radial arm maze ${ }^{13}$ and spatial reference memory in the Morris water maze (MWM) ${ }^{14}$. In addition, deficiencies in dietary folic acid and DNA repair exacerbates memory impairments in chronically hypoperfused mice ${ }^{15}$. The objective of this study was to determine whether increased levels of Hcy through deficiencies in MTHFR and dietary folic acid affect functional outcome in a mouse model of $\mathrm{VCl}$. Using a mouse model of $\mathrm{VCl}$ we report that a genetic and dietary deficiency in folate metabolism results in impaired reference memory, arterial remodeling along with reduced levels of acetylcholine and other choline metabolites.

\section{METHODS}

\subsection{Animal, diets and experimental design}

All experiments were approved by the Landesamt für Gesundheit und Soziales Berlin and performed in accordance with the German Animal Welfare Act. Generation of Mthfr deficient mice, was previously described ${ }^{10}$. The Mthfr null allele was backcrossed for more than 14 generations onto a C57BL/6 background ${ }^{16}$. Wild-type littermates as well as females and males from multiple litters were used for each group in all experiments. At four-weeks-of-age $\mathrm{Mthfr}^{+/+}$and $\mathrm{Mthfr}^{-/-}$mice were fed amino acid-defined diets containing 
amounts of nutrients recommended by the American Institute of Nutrition ${ }^{17}$. Mice were fed 1 of 2 diets: control (CD; 2 mg folic acid/kg diet, Envigo, Indianapolis, IN, USA) or folic acid deficient diet (FADD; $0.3 \mathrm{mg}$ folic acid/kg diet, Envigo, Indianapolis, IN, USA) All other nutrients and vitamins were not modified. Mice were maintained on these diets for six-weeks prior to surgery and remained so for the duration of the experiment (Figure 1). In order to remove neuroprotective effects of estrogen in females ${ }^{18}, 2$ weeks prior to microcoil implantation females underwent bilateral ovariectomy using dorsolateral procedure and males underwent a sham procedure with abdominal incisions. Afterwards animals were randomized into microcoil or sham control surgery groups using a custom written program (Mäuselotto). Animals, approximately 10 per group, were housed in a temperature $\left(22 \pm 2{ }^{\circ} \mathrm{C}\right)$, humidity $(55 \pm 10 \%)$, light $(12 / 12 \mathrm{~h}$ light/dark cycle) controlled environment, and given ad libitum access to food and water. Four weeks after surgery animals began a battery of behavioral tests, and approximately 6 weeks after surgery, MRI data was acquired. At the conclusion of the study, mice were deeply anaesthetized with isoflurane, terminal blood samples were taken and the brain was removed. The cortex was excised and frozen for gene expression, protein and choline metabolite analysis.

\subsection{Chronic hypoperfusion via bilateral common carotid artery stenosis}

Surgeries were carried out as previously described ${ }^{15}$. Briefly, animals were anaesthetized with isoflurane (1.5\%) and placed in a supine position. A midline incision exposed both carotid arteries and chronic hypoperfusion was induced by wrapping microcoils (180 $\mu \mathrm{m}$ inner diameter, Shannon Coiled Springs, Limerick, Ireland) around each artery ${ }^{12}$. The corresponding sham control procedure resulted in microcoils being 
placed next to the carotid arteries. All animals had local anesthetic applied to the wound after suturing the incision and received $6 \mathrm{mg} / \mathrm{mL}$ of Paracetamol in the drinking water one day prior to, and for three days after surgery, to assist with post-operative pain.

\subsection{Behavioural analysis}

\subsubsection{Rotarod}

Animals were tested on a single day in three separate trials as previously described ${ }^{15}$. Briefly, animals were placed on an accelerating rotarod (4 to $40 \mathrm{rpm}$ ) over $5 \mathrm{~min}$ and the latency to fall was recorded.

\subsubsection{Morris water maze}

Testing was carried out as previously described ${ }^{15}$. In brief, cues were present at fixed positions and a clear Plexiglas platform was submerged $1 \mathrm{~cm}$ beneath the surface of the water in the center of one of the four quadrants. The latency to find the platform for each animal was tracked with a computer-based system (TSE Systems, Bad Homburg vor der Höhe, Germany). Over 7 days mice were trained to find the hidden platform, with 3 trials per day and an inter-trial interval of $30 \mathrm{~min}$. Each trial consisted of a maximum of $90 \mathrm{~s}$ and was randomly started from one the quadrants with the animal facing the wall. If an animal did not reach the platform after $90 \mathrm{~s}$, it was guided there. On day 8 a probe trial (spatial probe) occurred, the platform was removed and mice were allowed to swim for $90 \mathrm{~s}$.

\subsubsection{Y-maze}


The maze was made of grey Plexiglas. Each mouse, naive to the maze, was placed at the end of one arm and allowed to move freely through the maze during an 8-min session. The series of arm entries was recorded. An arm entry was completed when the hind paws of the mouse had been completely placed in the arm; the number of entries per arm was recorded. Alternation was defined as successive entries into 3 arms, on overlapping triplet sets ${ }^{19}$. The percentage of alternation was calculated as the ratio of actual to possible alternations (defined as the total number of arms entered minus two), multiplied by $100^{20}$.

\subsection{Plasma Hcy levels}

At the completion of experiments, blood samples were collected via cardiac puncture and placed into potassium ethylenediaminetetraacetic acid (EDTA) coated tubes. Samples were centrifuged at $7000 \times \mathrm{g}$ for $7 \mathrm{~min}$ at $4^{\circ} \mathrm{C}$ to obtain the plasma and were subsequently frozen until analysis. Hcy concentrations were determined by high performance liquid chromatography (HPLC; Labor 28, Charité Medical University Berlin).

\subsection{Magnetic resonance imaging (MRI)}

All MRI experiments occurred 6 weeks post-operatively on a 7T system (Bruker BioSpin, Ettlingen, Germany) as previously described ${ }^{15}$. Briefly, a $20 \mathrm{~mm}$ diameter quadrature volume coil was used for radio frequency transmission and reception (RAPID Biomedical). The imaging protocol consisted of a $\mathrm{T}_{2}$ weighted 2D-rapid acquisition with relaxation enhancement (RARE) sequence (32 contiguous $0.5 \mathrm{~mm}$ thick slices, image matrix: 256 × 196 interpolated to 256 × 256, repetition time/echo spacing: TR/TE/ATE: $4200 / 36 / 12 \mathrm{~ms}$, RARE factor of 8,4 averages, resolution: $100 \mu \mathrm{m}^{2}, 6: 43 \mathrm{~min}$ ), and a 3D 
time of flight (TOF) angiography sequence (image matrix: $256 \times 196 \times 128$ interpolated to $256^{3}$, TR/TE: $15 / 2.5 \mathrm{~ms}$, $\alpha$ : Flip angle: $20^{\circ}$, resolution: $\left.100 \mu \mathrm{m}^{3}, 6: 08 \mathrm{~min}\right)$.

\subsubsection{MRI image analysis}

Raw angiography data was exported into FSL (FMRIB, Oxford, UK) software and the FLIRT tool was used to co-register all data sets. The protocol for angiography analysis has been previously described ${ }^{15}$. The overall size of the circle of Willis (vasculature) and basilar artery were calculated from the binary image by counting the number of voxels with a value of 1 , and the volumes were corrected for co-registration using the determinant of the FSL transformation matrix.

\subsection{Western blotting}

Protein extracts from the cortices $(\sim 30 \mathrm{mg})$ were prepared, separated by SDS-PAGE and transferred to nitrocellulose membranes. Primary antibodies were choline acetyltransferase (ChAT; Millipore) and glyceraldehyde 3-phosphate dehydrogenase (GAPDH; Cell Signalling, Danvers, MA, USA). Secondary antibodies were horseradish peroxidase (HRP)-conjugated donkey anti-rabbit IgG (GE Healthcare) and HRPconjugated donkey anti-goat IgG (Santa Cruz Biotechnology, Dallas, TX, USA), as appropriate. Detection was achieved using Western Lighting Plus ECL (Perkin Elmer, Waltham, MA. USA). Bands were quantified by densitometry using freeware ImageJ (National Institutes of Health) and normalized to GAPDH. Two experiments were performed for all cortical protein levels. 


\subsection{Choline metabolites}

Cortical acetylcholine, betaine, choline, glycerophosphocholine, phosphocholine, phosphatidylcholine and sphingomyelin were measured by the LC-MS method as previously reported ${ }^{21}$.

\subsection{Statist ${ }^{22-24}$ ical analysis}

Two investigators, blinded to group assignment, performed analysis of all behavioral data, gene expression and Western blot experiments. Statistical analysis was performed using Graph Pad 6.0 or SPSS 21 software. A two-way analysis of variance (ANOVA) was used to compare both the diet and surgery groups within each genotype. Two way repeated measure ANOVA was used for the Morris water maze daily latency measurements. Significant main effects in the ANOVAs were followed by the Bonferroni post-hoc test to determine whether statistically significant differences existed between the groups. In all analyses, $p \leq 0.05$ was considered significant. All data are presented as mean \pm standard error of the mean (SEM).

\section{RESULTS}

\subsection{No balance and coordination impairments in microcoil mice}

Prior to testing mice on the Morris water maze to determine whether motor function was impaired as a result of microcoil implantation, sensorimotor function was assessed using the rotarod ${ }^{22-24}$. No significant main effects for surgery (Figure $2 \mathrm{~A} ; \mathrm{Mthfr}^{+/+} \mathrm{F}(1,37)=$ $0.041, p=0.84$; Figure 2B, Mthfr $\left.{ }^{+/-} F(1,37)=0.29, p=0.59\right)$ nor $\operatorname{diet}\left(M\right.$ thfr $^{+/+} F(1,37)=$ $8.14 \times 10-5, p=0.99 ;$ Mthfr $^{+/-}(F(1,37)=0.40, p=0.53)$ were present. 


\subsection{Reference memory impaired in $\mathrm{Mthfr}^{+/+}$on FADD}

$\mathrm{Mthfr}^{+/+}$(Figure 3A) and Mthfr ${ }^{+/-}$(Figure 3B) mice learned the location of the platform during the 7 days of testing. Although when reference memory was assessed, there was a no differences were observed between $\mathrm{Mthfr}^{+/+}$mice (Figure 6C) but a significant diet effect was observed for $\mathrm{Mthfr}^{+/}$mice (Figure 3D; F $\left.(1,36)=4.7, \mathrm{p}<0.05\right)$. More specifically, FADD microcoil mice spent less time in the platform quadrant when compared to $C D$ sham $(p<0.01)$, FADD sham $(p<0.01)$ and FADD microcoil $(p<0.01)$ mice.

\subsection{No disturbances in spatial memory in microcoil mice}

To measure spatial short-term memory in mice we used the Y-maze task ${ }^{19}$. There was no diet or surgery effect on the number of alternations, visits or percent alternations made in the Y-maze within experimental groups for $\mathrm{Mthfr}^{+/+}$and $\mathrm{Mthfr}^{+/-}$mice (Table 2).

\subsection{Increased concentration of plasma Hcy in FADD $\mathrm{Mthfr}^{+/+}$and $\mathrm{Mthfr}^{+/-}$mice}

Plasma Hcy levels were measured at the competition of experiments; there was a significant diet main effect in $\mathrm{Mthfr}^{+/+}($Figure 4A, F $(1,33)=5.33, \mathrm{p}<0.05)$ and $\mathrm{Mthfr}^{+/-}$ (Figure 4B, $F(1,30)=18.04, p<0.001)$ mice. FADD consistently resulted in higher plasma homocysteine levels, but was more pronounced in the $\mathrm{Mthfr}^{+-}$mice. FADD microcoil and $M$ thfr $^{+/-}$FADD microcoil mice had significantly higher plasma homocysteine levels when compared to CD microcoil $(p<0.05)$ and CD sham mice $(p<0.05)$.

\subsection{Arterial remodeling in chronically hypoperfused $M t^{\prime h f r}{ }^{+/}$mice}


Representative images of the basilar artery and circle of Willis (vasculature) of $\mathrm{Mthfr}^{+/-}$ mice are shown in Figure $5 \mathrm{~A}$ to $\mathrm{D}$. A significant interaction between diet and surgery was observed in volume of the basilar artery (Figure 5E, $F(1,40)=8.49, p<0.05$ ) and vasculature (Figure 5F, F $(1,40)=4.54, p<0.01)$. Mthfr $^{+/}$FADD microcoil mice had significantly reduced basilar artery volume when compared to CD microcoil $(p<0.05)$ and FADD sham $(p<0.01)$ mice as a result of diet and surgery. Mthfr ${ }^{+/-}$FADD microcoil mice had reduced vasculature volume when compared to FADD sham $(p<0.05)$. No differences in $\mathrm{Mthfr}^{+/+}$groups were observed.

\subsection{Cortical choline metabolism altered with MTHFR-deficiency and chronic hypoperfusion}

Choline metabolism was measured in brain tissue to assess impact on this animal model. Cortical acetylcholine levels were reduced as a result of both diet and surgery in $\mathrm{Mthfr}^{+/+}$ (Table 1, F (1, 22) $=4.32, p<0.05)$ and diet in Mthfr $^{+/-}($Table 1, F $(1,20)=12.53, p<0.001)$ mice. No changes in betaine and choline were observed between any groups (Table 1). Phosphocholine levels were reduced in $\mathrm{Mthfr}^{+/+}$FADD mice $($Table $1, F(1,20)=6.78$, $p<0.05)$ with a decrease between CD and FADD microcoil mice $(p<0.05)$. Sphingomylein levels were reduced in Mthfr ${ }^{+/-}$FADD sham and microcoil mice (Table 1, $F\left({ }_{1,21}\right)=6.00$, $\mathrm{p}<0.05)$.

Choline acetyltransferase (ChAT) is another enzyme involved in metabolism of acetylcholine, specifically in the generation of acetylcholine at synapses. Cortical protein levels of ChAT were increased as a result of diet in the $M$ thfr $^{+/+}$mice (Figure 6A, F (1, 16) $=6.13, \mathrm{p}<0.05)$. In $\mathrm{Mthfr}^{+-}$mice there was a diet and surgery interaction (Figure 6B, F (1, $\left.\left.{ }_{16}\right)=10.37, p<0.01\right)$. Mthfr ${ }^{+/-}$FADD microcoil mice had significantly elevated levels of 
ChAT protein expression compared to CD sham $(p<0.05)$, FADD sham $(p<0.05)$ and CD microcoil $(p<0.05)$ mice.

\section{DISCUSSION}

Epidemiological research has linked deficiencies in folic acid metabolism and resultant elevated levels of Hcy to the development of $\mathrm{VCl}$, but the association remains controversial. The present study investigated the impact of elevated levels of Hcy via deficiencies in MTHFR and dietary folic acid on a mouse model of $\mathrm{VCl}$. No motor function deficits were observed between groups confirming that performance on Morris water maze (MWM) was not affected. When reference memory was assessed using the MWM, we observed that FADD $\mathrm{Mthfr}^{+/-}$microcoil mice spent less time in the platform quadrant. There was no impairment in spatial short-term memory using one trial y-maze task. Others studies have reported differences mice with microcoil implantation show impairments in spatial memory ${ }^{13,25}$. We confirmed that deficiencies in dietary folic acid and MTHFR resulted in increased levels of plasma homocysteine. We confirmed that deficiencies in dietary folic acid and MTHFR resulted in increased levels of plasma homocysteine. The data from this study suggests that hyperhomocysteinemia, via dietary folic acid combined with MTHFR-deficiency, and chronic hypoperfusion interact to reduce volumes of both the basilar artery and circle of Willis (vasculature), possibly as a result of disrupted endothelial cell function ${ }^{4}$. Interestingly, there was no arterial remodeling observed in wild-type mice suggesting that indeed MTHFR deficiency and chronic hypoperfusion combined change vasculature in chronically hypoperfused mice. For the

first time in a mouse model of $\mathrm{VCl}$ we showed changes in cortical choline metabolism, specifically levels of acetylcholine were reduced in chronically hypoperfused FADD mice 
and that similar changes were observed in related metabolites and enzymes that metabolize the neurotransmitter.

Patients affected by VCI have a reduction of $40 \%$ of cholinergic activity in brain tissue ${ }^{26}$. The impact of chronic hypoperfusion (microcoil) on choline metabolism in brain tissue has not been investigated. In the present study we are the first show that choline metabolism in cortical tissue was affected as a result of both chronic hypoperfusion and deficiencies in folate metabolism. Choline and folate metabolism are tightly linked ${ }^{27}$, especially in the brain ${ }^{28}$. Furthermore, the nutrient choline is essential in the generation of acetylcholine, a neurotransmitter involved in learning and memory ${ }^{29}$. A MTHFR deficiency has previously been shown to alter choline metabolism in brain tissue ${ }^{28,30}$. In the present study we report a combined effect of MTHFR deficiency and chronic hypoperfusion reduced ChAT protein levels in cortical tissue. ChAT levels have been shown to increase in response to low levels of acetylcholine ${ }^{31}$. Changes in acetylcholine levels did parallel impairments in memory function on the MWM for $\mathrm{Mthfr}^{+/-}$mice. Mthfr ${ }^{+/-}$ FADD microcoil mice spent significantly less time in the platform quadrant during the probe trial. Therapies targeting the cholinergic system have been targeted towards patients affected by $\mathrm{VCl}$. These therapies include cholinesterase inhibitors which have been reported to have minimal effects on cognitive impairment ${ }^{32,33}$. Results from the present study suggest that dietary choline supplementation may result in therapeutic benefit for $\mathrm{VCl}$ patients.

We show that elevated levels of homocysteine, through deficiencies in MTHFR and dietary folic acid, may indeed make animals more susceptible to vascular cognitive impairment. Furthermore, increasing levels of plasma Hcy through dietary folic acid 
deficiencies have been proposed as an animal model of vascular dementia ${ }^{34,35}$. However, the results from previous work suggests that increased levels of Hcy may not be the cause of the $\mathrm{VCl}^{15}$, but that they might be marker for disease state. Elevated levels of Hcy reflect changes in the other determinants ${ }^{36}$. For example, elevated levels of Hcy can be a result of dietary folic acid deficiencies. Folic acid plays an important role in nucleotide synthesis as well as DNA repair and we have previously shown that deficiencies in DNA repair and dietary folic acid in combination with chronic hypoperfusion lead to impaired learning and memory ${ }^{15}$. On the other hand, increased levels of Hcy increase could change homeostasis in the brain, that may increase susceptibility to neurological damage, such as increasing oxidative stress ${ }^{37}$. The present study brings to light that deficiencies in MTHFR when combined with dietary folic acid increase susceptibility to memory impairment in a mouse model of $\mathrm{VCl}$ through arterial remodeling and reduced levels of acetylcholine.

\section{CONCLUSION}

The data from the present study suggests that increased Hcy levels via deficiencies in MTHFR and dietary folic acid affect reference memory, vasculature and choline metabolism in a mouse model of $\mathrm{VCl}$. Future assessment of whether reducing levels of Hcy levels via choline supplementation may be beneficial for individuals with MTHFR deficiency may be worthwhile when designing therapeutic options.

\section{ACKNOWLEDGEMENTS}

We thank Dr. Rima Rozen for the gift of the Mthfr knockout mice, Susanne Mueller for assistance with MRI data collection and Anthony Carter for assistance with microcoil 
surgeries. Work was supported by BMBF (01EW1201) under the ERANET NEURON scheme (TDF) and the Fonds de la recherché en santé Québec, Canada (NMJ).

\section{REFERENCES}

1. Iadecola C. The Pathobiology of Vascular Dementia. Neuron. 2013;80:844-866.

2. O'Brien JTO, Erkinjuntti T, Reisberg B, Roman G, Sawada T, Pantoni L, et al. Vascular cognitive impairment. Lancet Neurol. 2003;2:89-98.

3. Perez L, Heim L, Sherzai a, Jaceldo-Siegl K. Nutrition and vascular dementia. J. Nutr. Health Aging. 2012;16:319-24.

4. Castro R, Rivera I, Blom HJ, Jakobs C, Tavares de Almeida I. Homocysteine metabolism, hyperhomocysteinaemia and vascular disease: an overview. J. Inherit. Metab. Dis. 2006;29:3-20.

5. Whalley LJ, Duthie SJ, Collins AR, Starr JM, Deary IJ, Lemmon H, et al. Homocysteine, antioxidant micronutrients and late onset dementia. Eur. J. Nutr. 2013;53:277-85.

6. Wald DS, Kasturiratne A, Simmonds M. Serum homocysteine and dementia: Metaanalysis of eight cohort studies including 8669 participants. Alzheimer's Dement. 2011;7:412-417.

7. Sharma M, Tiwari M, Tiwari RK. Hyperhomocysteinemia: Impact on Neurodegenerative Diseases. Basic Clin. Pharmacol. Toxicol. 2015;117:n/a-n/a.

8. Schneider JA, Rees DC, Liu YT, Clegg JB. Worldwide distribution of a common methylenetetrahydrofolate reductase mutation. Am. J. Hum. Genet. 1998;62:125860.

9. Frosst P, Blom HJ, Milos R, Goyette P, Sheppard CA, Matthews RG, et al. A candidate genetic risk factor for vascular disease: a common mutation in methylenetetrahydrofolate reductase. Nat. Genet. 1995;10:111-3.

10. Chen Z, Karaplis AC, Ackerman SL, Pogribny IP, Melnyk S, Lussier-Cacan S, et al. Mice deficient in methylenetetrahydrofolate reductase exhibit hyperhomocysteinemia and decreased methylation capacity, with neuropathology and aortic lipid deposition. Hum. Mol. Genet. 2001;10:433-43.

11. Jiwa NS, Garrard P, Hainsworth AH. Experimental models of vascular dementia and vascular cognitive impairment: a systematic review. J. Neurochem. 2010;115:814-28.

12. Shibata M, Ohtani R, Ihara M, Tomimoto $\mathrm{H}$. White matter lesions and glial activation in a novel mouse model of chronic cerebral hypoperfusion. Stroke. 2004;35:2598-2603.

13. Shibata M, Yamasaki N, Miyakawa T, Kalaria RNN, Fujita Y, Ohtani R, et al. Selective impairment of working memory in a mouse model of chronic cerebral hypoperfusion. Stroke. 2007;38:2826-2832. 
14. Füchtemeier M, Brinckmann MP, Foddis M, Kunz A, Po C, Curato C, et al. Vascular change and opposing effects of the angiotensin type 2 receptor in a mouse model of vascular cognitive impairment. J. Cereb. Blood Flow Metab. 2015;35:476-484.

15. Jadavji N, Farr T, Khalil A, Boehm-Sturm P, Foddis M, Harms C, et al. Elevated levels of plasma homocysteine, deficiencies in dietary folic acid and uracil-DNA glycosylase impair learning in a mouse model of vascular cognitive impairment. Behav. Brain Res. 2015;283:215-226.

16. Lawrance AK, Racine J, Deng L, Wang X, Lachapelle P, Rozen R. Complete deficiency of methylenetetrahydrofolate reductase in mice is associated with impaired retinal function and variable mortality, hematological profiles, and reproductive outcomes. J. Inherit. Metab. Dis. 2011;34:147-57.

17. Reeves PG, Nielsen FH, Fahey GC. AIN-93 purified diets for laboratory rodents: final report of the American Institute of Nutrition ad hoc writing committee on the reformulation of the AIN-76A rodent diet. J. Nutr. 1993;123:1939-51.

18. Roof RL, Hall ED. Gender differences in acute CNS trauma and stroke: neuroprotective effects of estrogen and progesterone. J. Neurotrauma. 2000;17:367-88.

19. Lalonde R. The neurobiological basis of spontaneous alternation. Neurosci. Biobehav. Rev. 2002;26:91-104.

20. Maurice T, Hiramatsu M, Itoh J, Kameyama T, Hasegawa T, Nabeshima T. Behavioral evidence for a modulating role of sigma ligands in memory processes. I. Attenuation of dizocilpine (MK-801)-induced amnesia. Brain Res. 1994;647:44-56.

21. Koc H, Mar MH, Ranasinghe A, Swenberg JA, Zeisel SH. Quantitation of choline and its metabolites in tissues and foods by liquid chromatography/electrospray ionization-isotope dilution mass spectrometry. Anal. Chem. 2002;74:4734-4740.

22. Brooks SP, Pask T, Jones L, Dunnett SB. Behavioural profiles of inbred mouse strains used as transgenic backgrounds. I: motor tests. Genes, Brain Behav. 2004;3:206-215.

23. Carter RJ, Morton J, Dunnett SB. Motor coordination and balance in rodents. Curr. Protoc. Neurosci. 2001; Chapter 8:Unit 8.12.

24. JONES BJ, ROBERTS DJ. The quantitative measurement of motor inco-ordination in naive mice using an accelerating rotarod. J. Pharm. Pharmacol. 1968;20:302304.

25. Coltman R, Spain A, Tsenkina Y, Fowler JH, Smith J, Scullion G, et al. Selective white matter pathology induces a specific impairment in spatial working memory. Neurobiol. Aging. 2011;32:2324.e7-12.

26. Erkinjuntti T, Román G, Gauthier S, Feldman H, Rockwood K. Emerging Therapies for Vascular Dementia and Vascular Cognitive Impairment. Stroke. 2004;35:10101017. 
27. Caudill MA. Folate and choline interrelationships: metabolic and potential health implications. In: Bailey LB, editor. Folate in Health and Disease. Boca Raton: CRC Press; 2009. p. 449-465.

28. Jadavji NM, Deng L, Leclerc D, Malysheva O, Bedell BJ, Caudill MA, et al. Severe methylenetetrahydrofolate reductase deficiency in mice results in behavioral anomalies with morphological and biochemical changes in hippocampus. Mol. Genet. Metab. 2012;106:149-59.

29. Gandhi CC, Kelly1 RM, Wiley RG, Walsh TJ. Impaired acquisition of a Morris water maze task following selective destruction of cerebellar purkinje cells with OX7saporin. Behav. Brain Res. 2000;109:37-47.

30. Chan A, Tchantchou F, Graves V, Rozen R, Shea TB. Dietary and genetic compromise in folate availability reduces acetylcholine, cognitive performance and increases aggression: critical role of S-adenosyl methionine. J. Nutr. Health Aging. 2008;12:252-61.

31. Wurtman RJ. Choline metabolism as a basis for the selective vulnerability of cholinergic neurons. Trends Neurosci. 1992;15:117-22.

32. Levine DA, Langa KM. Vascular Cognitive Impairment: Disease Mechanisms and Therapeutic Implications. Neurotherapeutics. 2011;8:361-373.

33. Parnetti L, Mignini F, Tomassoni D, Traini E, Amenta F. Cholinergic precursors in the treatment of cognitive impairment of vascular origin: Ineffective approaches or need for re-evaluation? J. Neurol. Sci. 2007;257:264-269.

34. Sudduth TL, Powell DK, Smith CD, Greenstein A, Wilcock DM. Induction of hyperhomocysteinemia models vascular dementia by induction of cerebral microhemorrhages and neuroinflammation. J. Cereb. Blood Flow Metab. 2013;33:708-15.

35. Troen AM, Shea-Budgell M, Shukitt-Hale B, Smith DE, Selhub J, Rosenberg IH. Bvitamin deficiency causes hyperhomocysteinemia and vascular cognitive impairment in mice. Proc. Natl. Acad. Sci. U. S. A. 2008;105:12474-9.

36. Nilsson K, Gustafson L, Hultberg B. Elevated plasma homocysteine level in vascular dementia reflects the vascular disease process. Dement. Geriatr. Cogn. Dis. Extra. 2013;3:16-24.

37. Köseoglu E, Karaman Y. Relations between homocysteine, folate and vitamin B12 in vascular dementia and in Alzheimer disease. Clin. Biochem. 2007;40:859-863. 


\section{FIGURES}

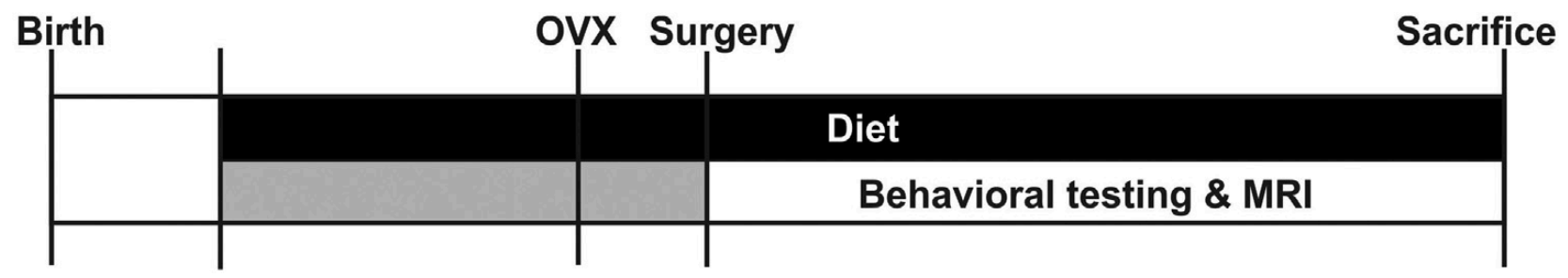

Figure 1. Experimental timeline of manipulations and outcome measurements. At 4 weeks of age mice were placed on control (CD) and folic acid deficient (FADD) diets, animals remained on these diets for the duration of experiment. Females underwent ovariectomy (OVX) and males the sham control procedures 2 weeks prior to implantation of microcoils. Three weeks after microcoil implantation animals were tested on the Morris water maze, Y-maze, and rotarod tasks. MRI measurements included volumetry and MRI angiography, at 5 weeks after microcoil implantation. At the completion of experiments brain tissue and plasma were collected for analysis.


Figure 2. The impact of MTHFR-deficiency, hyperhomocysteinemia and chronic hypoperfusion on balance and coordination on the rotarod task. Latency to fall off accelerating rotarod in $\mathrm{Mthfr}^{+/+}(\mathrm{A})$ and $\mathrm{Mthfr}^{+/-}$(B) mice. Depicted are means \pm SEM of ten to thirteen mice per group. No difference was observed between groups. 

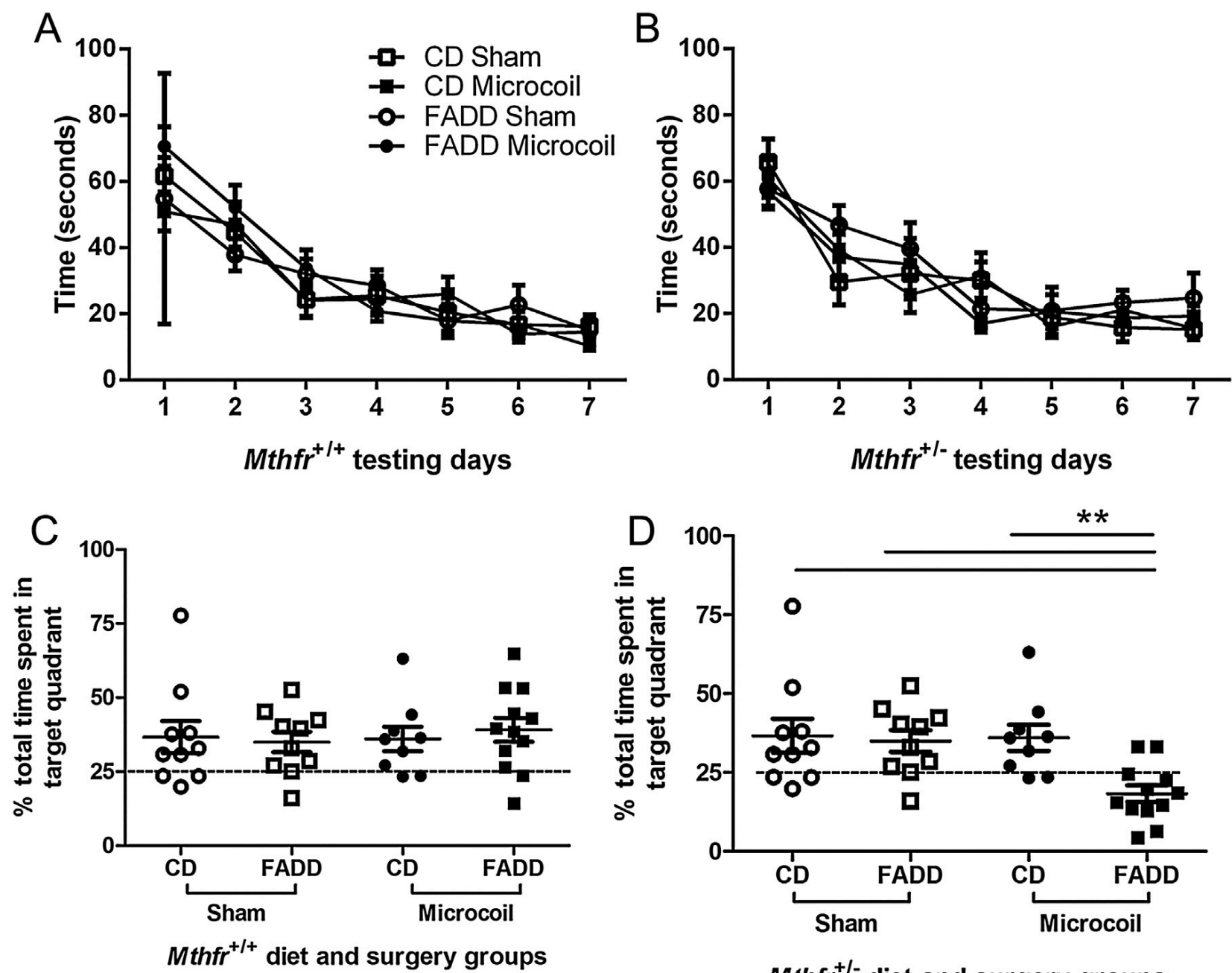

Mthfr $^{+/-}$diet and surgery groups

Figure 3. The effects of MTHFR-deficiency, hyperhomocysteinemia and chronic hypoperfusion on spatial learning and memory. Morris water maze escape latencies in $\mathrm{Mthfr}^{+/+}(\mathrm{A})$ and $\mathrm{Mthfr}^{+/-}$(B) mice. Percent time spent in platform quadrant (scatter plot of nine to thirteen mice per group) during probe trial on day 8 in $\mathrm{Mthfr}^{+/+}$(C) and $\mathrm{Mthfr}^{+/-}$(D) mice. ${ }^{*}$ indicates $p \leq 0.05$, Bonferroni pairwise post-hoc test. 



Figure 4. Plasma Hcy concentration in chronically hypoperfused $M t^{2} f^{+/}(\mathrm{A})$ and $M$ thfr $^{+/-}$ (B) mice on folic acid deficient (FADD) and control diets (CD). Depicted are means \pm SEM of seven to twelve mice per group. * indicates $p \leq 0.05$, Bonferroni pairwise posthoc test. 

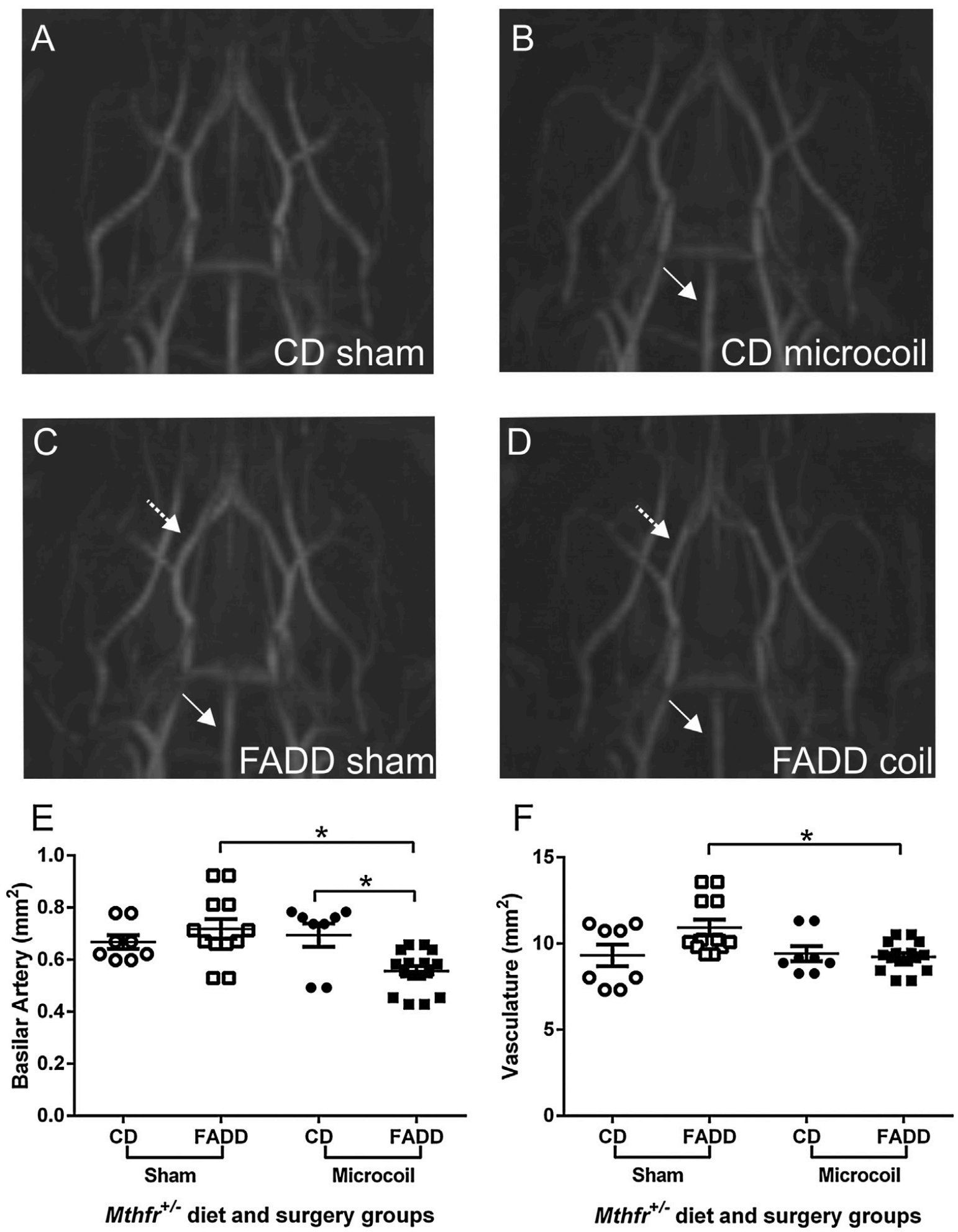

Figure 5. Representative angiography images of the basilar artery and circle of Willis in $\mathrm{Mthfr}^{+-}$mice maintained on CD (A) sham, (B) microcoil, and a FADD (C) sham, (D) microcoil. Solid arrows indicate differences in basilar artery and dashed arrows indicate vasculature (circle of Willis) differences between groups. Size of the basilar artery (E) and the overall circle of Willis vasculature $(F)$. Depicted are means \pm SEM of nine to thirteen mice per group. * indicates $p \leq 0.05$, Bonferroni pairwise post-hoc test. 

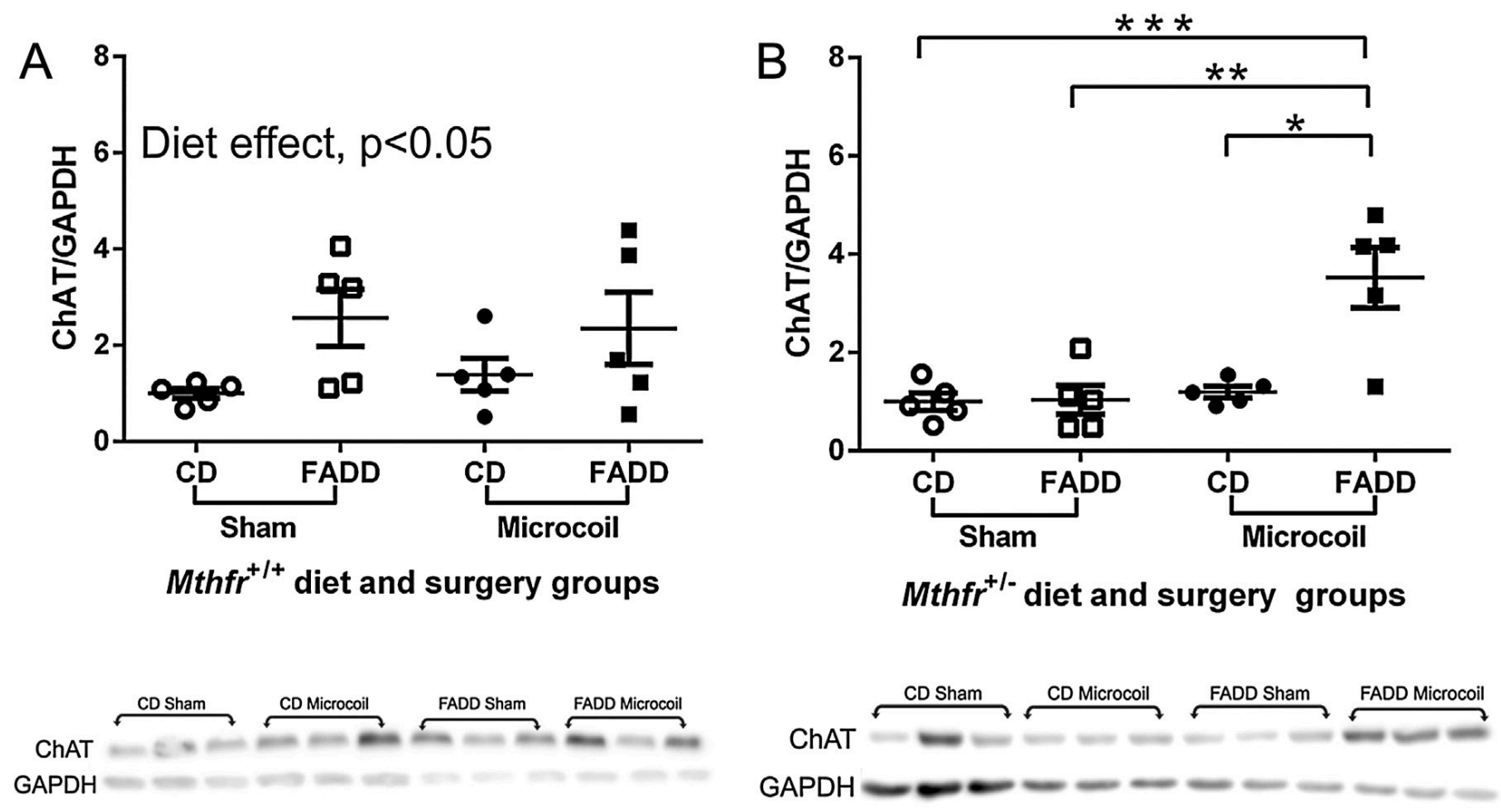

Figure 6. Protein levels of choline acetyltransferase (ChAT) in chronically hypoperfused cortex in $\mathrm{Mthfr}^{+/+}(\mathrm{A})$ and $\mathrm{Mthfr}^{+/-}(\mathrm{B})$ maintained on $\mathrm{CD}$ and $\mathrm{FADD}$. A representative Western Blot below histogram in (A) and (B). Depicted are means \pm _SEM of four to five mice per group. ${ }^{*}$ indicates $p \leq \_0.05,{ }^{* *}$ indicates $p \leq \_0.01$ and ${ }^{* * *}$ indicates $p<0.001$, Bonferroni pairwise post-hoc test. 
Table 1. Concentration of choline metabolites in cortical tissue of $\mathrm{Mthfr}^{+/+}$and $\mathrm{Mthfr}^{+/-}$on $\mathrm{CD}$ and FADD in microcoil and sham control mice. ${ }^{a}$

\begin{tabular}{|c|c|c|c|c|c|}
\hline \multirow[t]{2}{*}{ Diet and surgery groups } & \multicolumn{2}{|c|}{ Sham } & \multicolumn{2}{|c|}{ Microcoil } & \multirow[t]{2}{*}{$p$-value } \\
\hline & $\mathrm{CD}$ & FADD & $\mathrm{CD}$ & FADD & \\
\hline \multicolumn{6}{|c|}{ Genotype and metabolite $(\mathrm{nmol} / \mathrm{g})$} \\
\hline \multicolumn{6}{|c|}{ Mthfr ${ }^{+/ 4}$} \\
\hline Acetylcholine & $4.4 \pm 0.3$ & $3.4 \pm 0.2$ & $3.6 \pm 0.5$ & $3.8 \pm 0.4$ & $p<0.05^{b}$ \\
\hline Choline & $159.9 \pm 15.9$ & $156.0 \pm 18.1$ & $205.2 \pm 16.2$ & $144.8 \pm 7.0$ & n.s. \\
\hline Betaine & $11.0 \pm 0.5$ & $9.7 \pm 1.0$ & $17.4 \pm 0.8$ & $7.8 \pm 1.0$ & n.s. \\
\hline Sphingomylein & $5146 \pm 148.6$ & $5147 \pm 247.0$ & $5442 \pm 70.3$ & $5129 \pm 179.9$ & n.s. \\
\hline Phosphocholine & $451.0 \pm 14.3$ & $432.7 \pm 11.6$ & $460.1 \pm 17.6$ & $403.2 \pm 10.9$ & $p<0.05^{\mathrm{c}, e}$ \\
\hline Glycerophosphocholine & $761 . \pm 34.2$ & $734.2 \pm 54.7$ & $743.0 \pm 25.7$ & $716.2 \pm 24.0$ & n.s. \\
\hline \multicolumn{6}{|l|}{ Mthfr ${ }^{+/ 2}$} \\
\hline Acetylcholine & $4.6 \pm 0.5$ & $3.8 \pm 0.3$ & $4.6 \pm 0.4$ & $3 \pm 0.1^{d, e}$ & $p<0.05^{c}$ \\
\hline Choline & $154.8 \pm 15.3$ & $147.2 \pm 14.0$ & $164.9 \pm 19.5$ & $159.6 \pm 1.2$ & n.s. \\
\hline Betaine & $12.4 \pm 2.4$ & $6.3 \pm 0.3^{\prime}$ & $9.7 \pm 1.1$ & $7.4 \pm 1.1$ & $p<0.05^{c}$ \\
\hline Sphingomylein & $4893 \pm 125.5$ & $4602 \pm 98.4$ & $4850 \pm 98.4$ & $4635 \pm 90.3$ & $p<0.05^{c}$ \\
\hline Phosphocholine & $410.3 \pm 10.1$ & $411.1 \pm 12.5$ & $434.5 \pm 17.9$ & $401.5 \pm 12.8$ & n.s. \\
\hline Glycerophosphocholine & $826.8 \pm 24.5$ & $773.0 \pm 33.0$ & $804.4 \pm 26.1$ & $781.7 \pm 31.6$ & n.s. \\
\hline
\end{tabular}

${ }^{\text {a }}$ All values are means + SEM of 6 to 7 mice per group

${ }^{\mathrm{b}} \mathrm{p}$-value indicates significant diet and surgery interaction

${ }^{c} p$-value indicates dietary main effect

d $p$-value indicates significant Bonferroni pair wise comparison between CD Sham controls vs. FADD Microcoil

${ }^{e} p$-value indicates significant Bonferroni pair wise comparison between CD vs. FADD Microcoil

${ }^{f} p$-value indicates significant Bonferroni pair wise comparison between $C D$ and FADD Sham controls

Table 2. Functional behavioural outcome. Spatial memory (Y-maze) in $M t h f r^{+/+}$and $M$ thfr $^{+/-}$mice on folic acid deficient (FADD) and control (CD) diets with chronic hypoperfusion. ${ }^{a}$

\begin{tabular}{|c|c|c|c|c|c|c|c|c|}
\hline & \multicolumn{4}{|l|}{ Mthfr $\mathrm{r}^{+/+}$} & \multicolumn{4}{|l|}{ Mthfr ${ }^{+1-}$} \\
\hline & \multicolumn{2}{|l|}{$\mathrm{CD}$} & \multicolumn{2}{|l|}{ FADD } & \multicolumn{2}{|l|}{ CD } & \multicolumn{2}{|l|}{ FADD } \\
\hline & Sham & Microcoil & Sham & Microcoil & Sham & Microcoil & Sham & Microcoil \\
\hline Number of alternations & $7.6 \pm 1.1$ & $6.4 \pm 0.5$ & $6.7 \pm 0.6$ & $7.4 \pm 0.8$ & $6.9 \pm 0.7$ & $6.9 \pm 0.5$ & $7.8 \pm 0.6$ & $7.5 \pm 0.4$ \\
\hline Number of visits & $29.3 \pm 3.2$ & $25.8 \pm 1.7$ & $26.6 \pm 2.1$ & $29.4 \pm 2.0$ & $26.1 \pm 2.0$ & $27.5+2.0$ & $27.4+1.6$ & $28.1+0.9$ \\
\hline Percent alternation & $27.5 \pm 1.8$ & $26.5 \pm 1.2$ & $26.1 \pm 1.3$ & $27.9 \pm 1.3$ & $28.1 \pm 1.0$ & $30.5+1.0$ & $27.2+1.9$ & $28.6+1.0$ \\
\hline
\end{tabular}

a All values are means + SEM of 10-12 mice per group.

${ }^{a}$ All values are means \pm SEM of 10-12 mice per group 